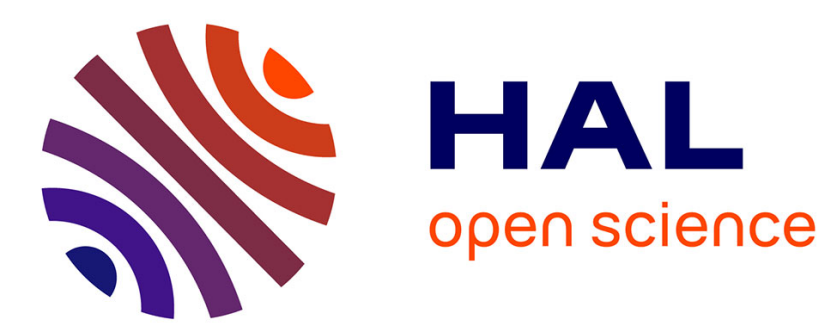

\title{
A compact dual polarized 3:1 bandwidth omnidirectional array of spiral antennas
}

Regis Guinvarc'H, Mohammed Serhir, Fabrice Boust

\section{To cite this version:}

Regis Guinvarc'H, Mohammed Serhir, Fabrice Boust. A compact dual polarized 3:1 bandwidth omnidirectional array of spiral antennas. IEEE Antennas and Wireless Propagation Letters, 2016, 15, pp.1909 - 1912. 10.1109/LAWP.2016.2542982 . hal-01309233

\section{HAL Id: hal-01309233 \\ https://hal.science/hal-01309233}

Submitted on 16 Sep 2016

HAL is a multi-disciplinary open access archive for the deposit and dissemination of scientific research documents, whether they are published or not. The documents may come from teaching and research institutions in France or abroad, or from public or private research centers.
L'archive ouverte pluridisciplinaire HAL, est destinée au dépôt et à la diffusion de documents scientifiques de niveau recherche, publiés ou non, émanant des établissements d'enseignement et de recherche français ou étrangers, des laboratoires publics ou privés. 


\title{
A compact dual polarized 3:1 bandwidth omnidirectional array of spiral antennas
}

\author{
Régis Guinvarc'h, Mohammed Serhir, and Fabrice Boust,
}

\begin{abstract}
A circularly polarized wideband omnidirectional array of spiral antennas is presented. It covers the frequency band from $1.2 \mathrm{GHz}$ up to $3.6 \mathrm{GHz}$ while keeping a good S11 and an omnidirectional coverage. This array achieved a dual polarization using left and right hands archimedean spiral antennas. The gain of the array is enhanced using a unique cavity for the entire array and the realized gain is above the maximum theoretical gain of a single spiral antenna.
\end{abstract}

Index Terms-Spiral antenna, wideband array, omnidirectional antennas, compact antenna, radar

\section{INTRODUCTION}

$\mathbf{O}$ MNI directional array can be used in various kind of applications, such as direction finding or passive radar. For most of these applications, it is desirable to get as much information as possible from the various azimuthal space directions. So a wide bandwidth and dual polarization capability are some interesting features. Whether it is for airborne, spaceborne or ground based uses, a compact system is a very good asset.

One way to build wideband arrays is to use wideband elements. Considering dual polarized omnidirectional arrays, this has been studied with horn antennas [1], bowtie etc.. Spiral antennas have been used in different kind of arrays: linear [2], planar [3], conformal [4] etc., but not in circular compact array, to the best of our knowledge. Its wide impedance bandwidth, along with its excellent polarization properties and wideband radiation pattern, makes it however an interesting candidate.

This paper describes a circular array of spiral antennas providing an omnidirectional coverage, over a wide frequency bandwidth. The dual polarization capability is ensured through the use of an alternate configuration. The array can emit (or collect) signals in any given direction, but not all the direction at the same time. The focus is put on the compactness so that it can be easily used in many applications. It is associated with a good gain thanks to the design of the array and in particular to the backing cavity. Considering passive applications (so positive gain as the criterion), the diameter of the antenna is only one wavelength at the lowest operating frequency. For active applications (so a reflection coefficient less then -10 $\mathrm{dB})$, a 3:1 bandwidth is guaranteed.

Section II describes the array configuration, along with the array element and the cavity design. Section III provides

R. Guinvarc'h is with SONDRA, CentraleSupelec, Universit Paris-Saclay, 91192 Gif-sur-Yvette, France (regis.guinvarch@centralesupelec.fr)

M. Serhir, is with GEEPS, UMR 8507 (CentraleSupelec - CNRS UPSud - UPMC) 11 rue Joliot-Curie 91192 Gif Sur Yvette Cedex - France

F. Boust is with SONDRA and ONERA - The French Aerospace Lab, Palaiseau, France

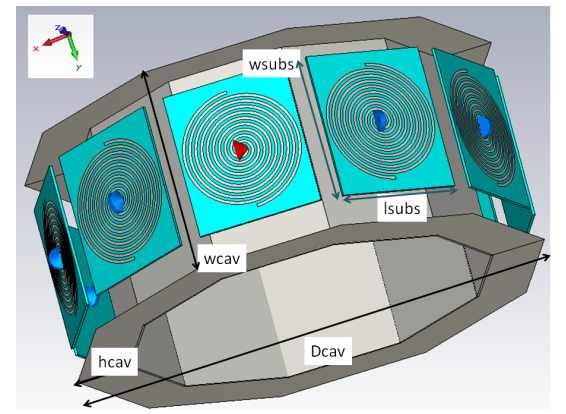

Fig. 1. The spiral antennas are in alternate configuration. hcav is $30 \mathrm{~mm}$, wcav is $122 \mathrm{~mm}$ and dcav is $280 \mathrm{~mm}$. lsusb is $72.4 \mathrm{~mm}$ and wsubs is $86 \mathrm{~mm}$.

measured and simulated results comparison. Finally, in Section IV concluding remarks are presented.

\section{Antenna DESIGN}

When designing a compact omnidirectional array of spiral antennas, there are two typical challenges. At low frequencies, the issue is the size of the antenna. Whatever the criteria (gain, polarization etc.), to reach lower frequencies, a spiral antenna has to get bigger. At the other end of the frequency range, the issue is the beamwidth of the spiral antenna to cover $360^{\circ}$.

\section{A. An Archimedean spiral antenna}

The Archimedean spiral antenna is a well known wideband antenna. Its lower operating frequency $f_{\min }$, given in Eq. 1, is inversely proportional to the antenna diameter $\mathrm{D}$. There has been a lot of work to miniaturize it, however, we will use its simplest version, so without any loading, meandering [5] etc.

$$
f_{\min }=\frac{c}{\pi D}
$$

\section{B. Spiral antennas omnidirectional array}

The omnidirectional feature requires $N$ spiral antennas placed regularly over a cylinder and facing different directions. The spiral antennas planes are orthogonal to the radii of the circle, as shown on Fig. 1. The resulting structure presents a rotational symmetry without any edge element. Hence, all the elements have the same properties. As for the design, the main parameter is the number of spiral antenna to be used in the array in order to cover the $360^{\circ}$ over the frequency bandwidth. The main difficulty occurs generally at the highest operating frequency. The spiral antenna with its broad beam was particularly interesting for this purpose. 


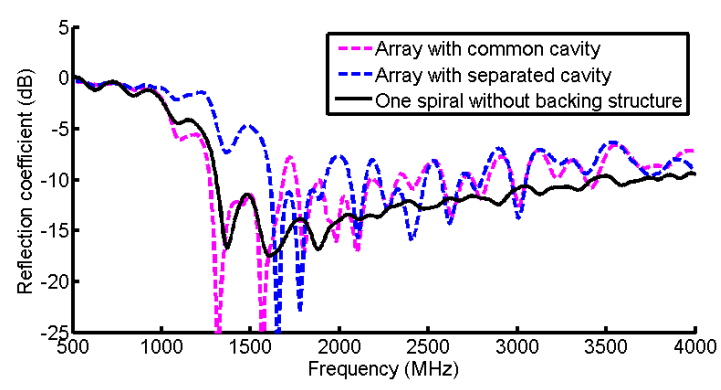

Fig. 2. Simulated reflection coefficients for common and separated cavities.

The minimum circumference $(r)$ of the array is directly linked to the size of the spiral element $D$ and so to the lowest frequency of operation $f_{\min }$. The antenna array radius is linked to the number of the array spirals and to the spiral antenna diameter. We can easily infer:

$$
r>\frac{N D}{2 \pi}
$$

For instance, a 5-spiral antenna array implies a $72^{\circ}$ beamwidth to cover $360^{\circ}$. From simulations of single spiral in free space, Eq. (2) leads to a maximum value of $3.4 \mathrm{GHz}$ for a single $72 \mathrm{~mm}$ spiral, with a minimum radius of $5.7 \mathrm{~cm}$. Of course, this does not take into account the coupling between the array element and with the cavity. This is studied later with the simulation of the full structure.

\section{Polarization}

Spiral antennas of both left and right hand circular polarization have to be used in alternate configuration to get a dual polarization capability. The number of elements $N$ has then to be doubled to account for this alternate configuration. Consequently, the minimum number of antennas to be used to get dual polarization with the omnidirectional feature is 10 . Using a spiral antenna with a diameter of $72 \mathrm{~mm}$, this gives the smallest possible array, with a radius of $11.5 \mathrm{~cm}$.

\section{Cavity design and gain improvement}

The ground plane has been shaped to follow the decagon shape. Two metallic sides have been added to create a cavity. This ensures an unidirectional beam, with lots of space in the middle of the antenna to put components.

Besides the unidirectional feature, the cavity plays also an important role for the gain enhancement. The gain is generally limited by the size of the antenna aperture, this is particularly important at low frequencies. Using the proposed cavity design, we have created an infinite cavity along the array dimension. It allows the field to spread in a larger space. At high frequency, the current does not reach the end of the arms, so there is no difference. At low frequency, instead of being reflected at the end of the arm, the current can couple onto the next spiral through some kind of transmission line with the ground. $S_{2,1}$ reaches $-12 \mathrm{~dB}$ at $1.25 \mathrm{GHz}$ and $1.5 \mathrm{GHz}$. This avoids the energy to be brought back to the generator (which would lead to a bad VSWR), while radiating the wrong

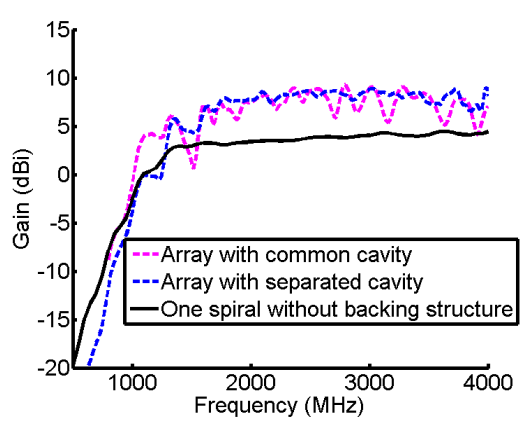

Fig. 3. Simulated gain for common and separated cavities.

polarization; on the contrary, the neighboring spiral being of the opposite polarization and fed at the end of its arm by coupling, it radiates the good polarization. This is illustrated in Fig. 2 with a comparison of the reflection coefficient of the array with a common cavity in dashed purple and with separated cavities (one per antenna) in dashed blue. The $S_{11}$ cutoff frequency has been lowered by about $350 \mathrm{MHz}$. In addition, Fig. 3 shows that the common cavity results in 4 $\mathrm{dB}$ gain improvement over this bandwidth, with a lower limit to get a positive gain at $1000 \mathrm{MHz}$ instead of $1250 \mathrm{MHz}$. As the gain improvement is above $3 \mathrm{~dB}$, it means a better matching of the antenna due to the design of the cavity.

From Fig. 3 additional oscillations for the common cavity are noticed on the gain, not on the reflection coefficient. This is due to reflections on the ground plane where it is not parallel to the antenna. It is worth noting that the principle of the common cavity is valid for many arrays made of spiral antennas. In [2], we introduced it for simple linear arrays with only 4 elements, the cavity being still of finite size. In [3], we extended the idea to a planar array of circular shape, where the cavity can already be seen as 1-D. We use here this principle, but with the array conformed to a decagone, with the antennas outwardly.

\section{MEASUREMEnTS RESUlts AND ANALysis}

We have built a prototype with $N=10$ elements. This is presented in Fig. 4 and 7. The cavity is made from copper and describes the decagone over which a foam material is placed (white part). Its dimensions are taken from [2] and are recalled in Fig. 1. This foam material present a permittivity near to 1 and is supposed to not disturb the radiating behavior of the spiral antennas. These 5 -turns spiral antennas are printed over FR4 dielectric substrate with $\epsilon_{r}=4.2$ of $1.6 \mathrm{~mm}$ thickness. Then the antennas are placed over the foam material. The top view of the antenna array prototype is presented in Fig. 4. In the middle of the photography we see the feeding cables associated to each antenna. Indeed, we used a pair of coaxial cable to excite symmetrically each spiral antenna. Two RG-10 coaxial cables are soldered together and the inner conductor of each cable is connected to the spiral arms. So the impedance between the two inner conductors (so seen from the antenna) is $100 \mathrm{ohms}$. The input impedance of the spirals when placed over a FR4 substrate with $1.6 \mathrm{~mm}$ thickness is $120 \Omega$ as presented in [2]. The two opposed-phase signals associated to each coaxial cable are generated using a $180^{\circ}$ 


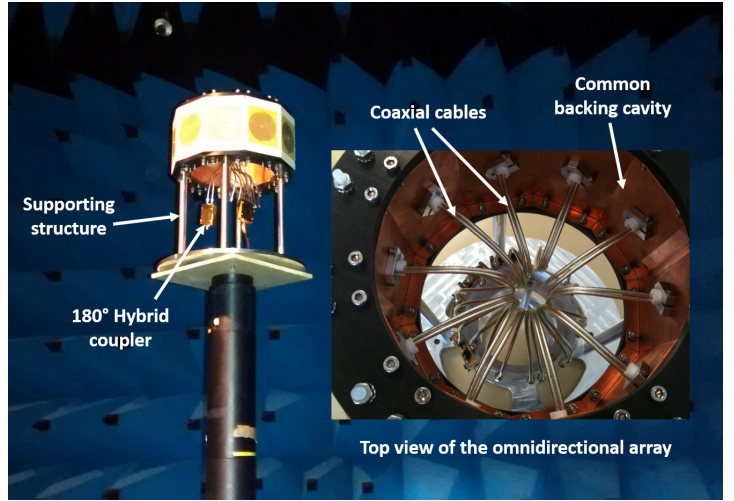

Fig. 4. Antenna in the anechoic chamber with an inset of the top view of the antenna, with the feeding cables paired to ensure the matching.

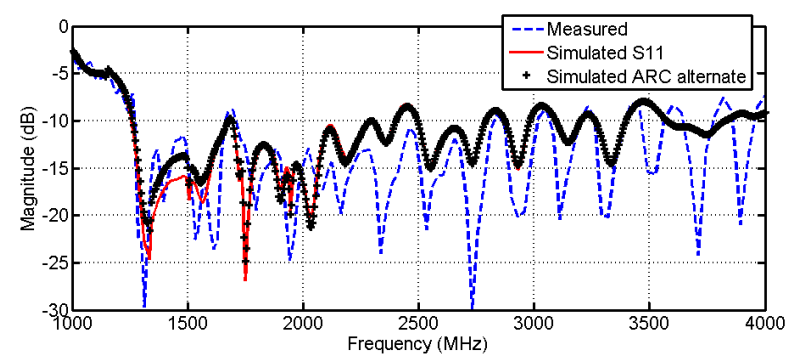

Fig. 5. Comparison between measured and simulated reflection coefficients of the prototype (without the matching structure losses). The active reflection coefficient (ARC) is also included.

hybrid coupler. This excitation technique can be generalized to any symmetrical structure with an input impedance around $100 \Omega$ [2]. The realized prototype has been measured in the CentraleSupelec measurement facility at the far-field distance over the frequency band $500 \mathrm{MHz}$ up to $4 \mathrm{GHz}$.

\section{A. Reflection coefficient}

The measured and the simulated reflection coefficients are plotted in Fig. 5. It must be mentioned that, thanks to the feeding structure depicted above, the reference impedance is $50 \Omega$. When not in an array, the reflection coefficient of the spiral antenna should be less than $-10 d B$ starting at $1.32 \mathrm{GHz}\left(\frac{c}{D \pi}\right)$. When placed in the array, the same spiral antenna presents a reflection coefficient $S 11$ below $-10 d B$ after $1.27 \mathrm{GHz}$. It is satisfactory up to at least $4 \mathrm{GHz}$. For the passive applications, it is interesting to consider that it may be usable starting around $1 \mathrm{GHz}$. There is a very good agreement between the measurement and the simulation, especially at low frequencies. The ground plane being at $3 \mathrm{~cm}$ from the antenna, the destructive interference should occur around 5 GHz. The active reflection coefficient [6] is also shown. It is very almost identical to the S11, except at low frequency where the maximum difference is only $2.5 \mathrm{~dB}$.

\section{B. Gain}

In Fig. 6 we compare the measured gain of the array and the simulated one including the losses of the feeding system. These losses are quite high (above $4 \mathrm{~dB}$ ) because of the large

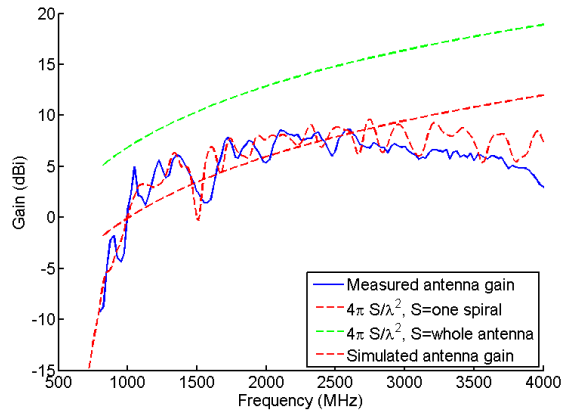

Fig. 6. Comparison between measured and simulated gain of the prototype (without the matching structure losses).

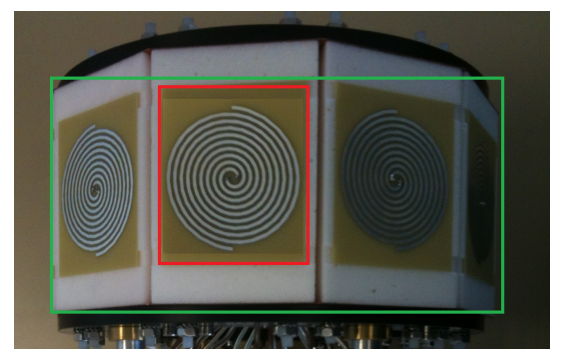

Fig. 7. Surfaces taken into account to calculate $\frac{4 \pi S}{\lambda^{2}}$.

losses in the hybrid couplers. However, these particular hybrids are interesting as they are quite small and as such, quite easy to include in the structure. Despite these high losses, the system gain is positive, except at the low frequency end. For passive radar at these bands, it is not an issue. There is a deep caused by a beam splitting around $1.5 \mathrm{GHz}$ in simulation orthogonally to the array axis. It is shifted in the measurements to $1.6 \mathrm{GHz}$ and almost canceled (less than $3 \mathrm{~dB}$, see Fig. 9). Along the array axis, the deep does not appear (see Fig. 8). In a first design, we used larger spirals of $78 \mathrm{~mm}$ [7]. The deep was much stronger and not canceled in the measurements.

The maximum expected gain $\frac{4 \pi S}{\lambda^{2}}$ is also plotted on Fig. 6 , for a surface $\mathrm{S}$ of the size of a single antenna substrate in red and for the projected surface of the whole antenna in green. The gain of the antenna is higher than the theoretical maximum gain of a single antenna on half of the bandwidth. It demonstrates the interest of the unique cavity to spread the field on the whole antenna and thus to improve the gain.

\section{Radiation patterns}

Fig. 8 shows the measured normalized radiation patterns for eight frequencies, from $1200 \mathrm{MHz}$ up to $3900 \mathrm{MHz}$, and are compared with the simulation results. Simulations and measurements show similar behavior. At broadside, the agreement is very good especially for low frequencies (see Fig. 6). However, out of the main beam there can be some discrepancies. This is probably due to fabrication inaccuracy and measurement uncertainties in the anechoic chamber (seen for higher frequencies), as well as some effect of the supporting structure. This was not taken into account in the simulation as the antenna array is too large to be simulated. In order to fulfill the $360^{\circ}$ coverage requirements, every pattern must 

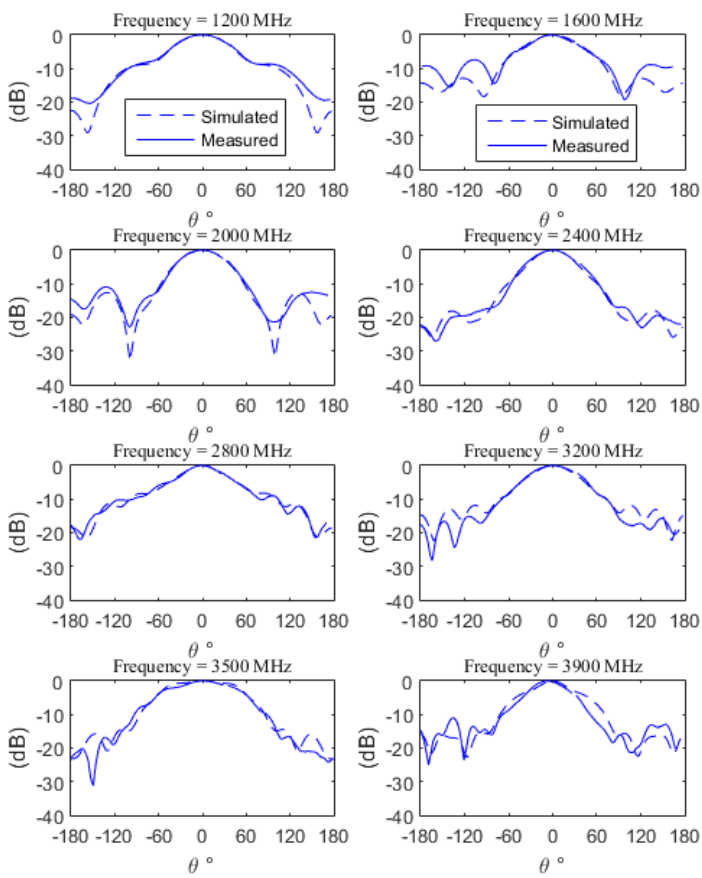

Fig. 8. Measured (solid lines) and simulated (dashed lines) radiation patterns for 8 frequencies, along the array axis (xz plane of Fig. 1).

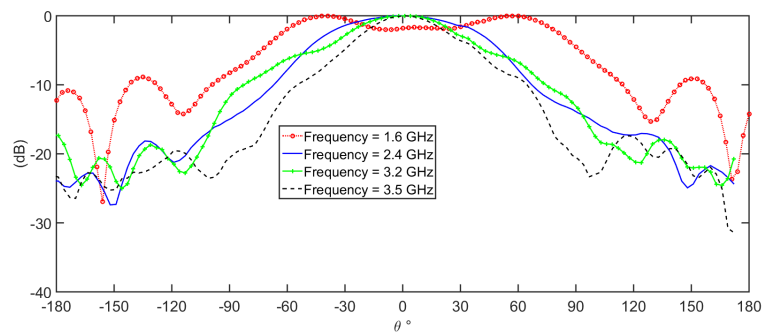

Fig. 9. Measured radiation patterns for 4 frequencies, orthogonal to the array axis (yz plane of Fig. 1).

have a $360^{\circ} / 5=72^{\circ} 3$-dB beamwidth in the $\phi=0^{\circ}$ plane, whatever the frequency. This was achieved for the simulation. But for the prototype, the criteria has to be lowered to a 4-dB beamwidth, which is still a good value given the compact size of the antenna. Fig. 9 plots the measured radiation patterns for four frequencies, orthogonally to the array axis. The beam is quite broad, typical of spiral antenna despite being narrower in this plane cut because of the sides of the cavity. It goes narrower as the frequency increases. At $1.6 \mathrm{GHz}$, the beam is slightly split. This corresponds to the loss of broadside gain at $1.6 \mathrm{GHz}$ of Fig. 6. So that loss is not due to a mismatching (as could also be seen on Fig. 5). There were no specific requirements over the radiation pattern in this plane.

\section{Cross polarization rejection}

The cross polarization rejection of Fig. 10 demonstrates a good agreement between simulation and measurement results. The cross polarization rejection is around $10 \mathrm{~dB}$, which is not a satisfactory value for many applications. It is particularly interesting to do the comparison with a single spiral antenna

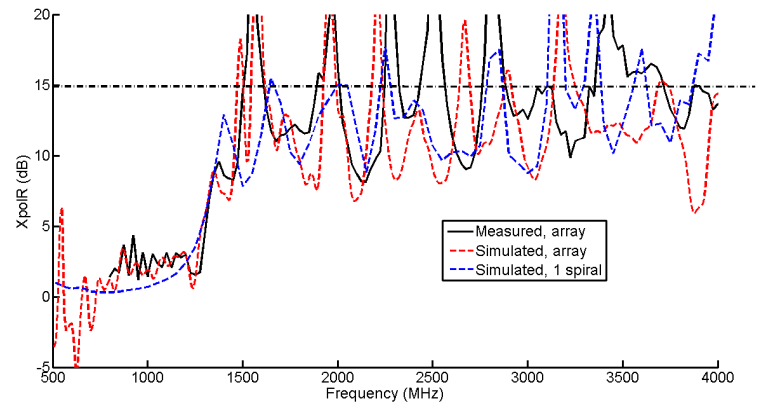

Fig. 10. Comparison between measured and simulated rejection of cross polarization rejection. The corresponding value for the spiral antenna alone above a ground plan is also indicated for reference. The horizontal dashed line at $15 \mathrm{~dB}$ indicates an Axial Ratio of $3 \mathrm{~dB}$.

above a ground plane (dashed blue line in Fig. 10). The values are roughly the same, in particular the low frequency behavior is absolutely not affected by the array, despite the conformal shape that could have been expected to alter the polarization. This leads to the conclusion that the array geometry, including the cavity, does not alter the polarization. As a consequence, the polarization could be improved by simply using optimized spirals with slow-wave techniques (see [5] for instance).

\section{CONCLUSion}

A compact wideband omnidirectional array of spiral antennas has been presented. It is almost one lambda of diameter at the lowest operating frequency. It works from $1.2 \mathrm{GHz}$ up to $3.6 \mathrm{GHz}$, with a good reflection coefficient and an omnidirectional coverage. The use of a unique cavity has increased the gain above the maximum theoretical gain of one antenna, in addition to put the cavity effects outside the bandwidth. It results in a positive gain from 1 (so below the theoretical cutoff frequency of the spiral) to at least $4 \mathrm{GHz}$.

\section{REFERENCES}

[1] Z. Wang, P. Hall, J. Kelly, and P. Gardner, "Tem horn circular array for wide band pattern notch reconfigurable antenna system," in Antennas and Propagation Conference (LAPC), 2010 Loughborough, Nov 2010, pp. 365-368.

[2] M. Serhir and R. Guinvarc'h, "A low-profile cavity-backed dual-polarized spiral antenna array," Antennas and Wireless Propagation Letters, IEEE, vol. 12, pp. 524-527, 2013.

[3] I. Hinostroza Saenz, R. Guinvarc'h, R. Haupt, and K. Louertani, "A dualpolarized wideband planar phased array with spiral antennas," Antennas and Propagation, IEEE Transactions on, vol. 62, no. 9, pp. 4547-4553, Sept 2014.

[4] F. Chauvet, R. Guinvarc'h, and M. Hélier, "Approximated method neglecting coupling for conformal array," Applied Computational Electromagnetics Society Journal, Special Issue on ACES 2006 Conference, pp. 105-111, March 2007.

[5] M. Lee, B. Kramer, C.-C. Chen, and J. Volakis, "Distributed lumped loads and lossy transmission line model for wideband spiral antenna miniaturization and characterization," Antennas and Propagation, IEEE Transactions on, vol. 55, no. 10, pp. 2671-2678, Oct 2007.

[6] H. Holter and H. Steyskal, "On the size requirement for finite phasedarray models," Antennas and Propagation, IEEE Transactions on, vol. 50, no. 6, pp. 836-840, Jun 2002.

[7] R. Guinvarc'h, M. Serhir, F. Boust, and N. Ribiere-Tharaud, "A wideband omnidirectional conformal array for passive radar," in Antennas and Propagation Society International Symposium (APSURSI), 2014 IEEE, July 2014, pp. 237-238. 